\title{
The better effects of microbubble ultrasound transfection of miR-940 on cell proliferation inhibition and apoptosis promotion in human cervical cancer cells
}

This article was published in the following Dove Press journal: OncoTargets and Therapy

\author{
Xiaojun Xiao' \\ Yujuan Zhang' \\ Qi Lin' \\ Keli Zhong ${ }^{2}$ \\ 'Department of Ultrasound, Shenzhen \\ People's Hospital, Shenzhen, Guangdong \\ Province 518020, People's Republic of \\ China; ${ }^{2}$ Department of Surgery, \\ Shenzhen People's Hospital, Shenzhen, \\ Guangdong Province 518020, People's \\ Republic of China
}

Correspondence: Keli Zhong Department of Surgery, Shenzhen People's Hospital, No.1017 Dongmen North Road, Luohu District, Shenzhen, Guangdong Province 518020, People's

Republic of China

$\mathrm{Tel}+8675525533018$

Email keli_zhokl@I63.com
Purpose: Cervical cancer is the second leading cause of women's cancer-related death. MiR-940 has been reported as a critical factor in various cancers. Based on the high transfection efficiency and low side effect, the clinical application of microbubble ultrasound contrast agent in gene treatment has attracted a widespread attention. In this study, we determined the mechanism of miR-940 inhibiting cell proliferation and cycle procession, and promoting cell apoptosis in cervical cancer Hela cells. In addition, we compared the effects of different transfection methods, including liposome, microbubble, ultrasound, and microbubble coupled with ultrasound.

Patients and methods: MTT assay, PI staining, and Annexin-V/PI staining assays were, respectively, performed to evaluate cell proliferation status, cell cycle progression, and apoptosis status. RT-PCR and Western blot were conducted to measure the levels of cell cycle- and apoptosis-related factors, and the phosphorylation levels of PI3K and Akt.

Results: Results showed that the overexpression of miR-940 inhibited cell proliferation, blocked cell cycle, and promoted apoptosis by regulating cell cycle-related factors (such as inhibited Cyclin D1 and CDK4) and apoptosis-related factors (such as promoted Puma and Bax, inhibited Bcl-2 and Cleaved caspase9), and inhibiting the phosphorylation and activation of PI3K/AKT pathway. Among all of them, miR-940 transfected with microbubble and ultrasound showed the greatest changes.

Conclusion: It provides evidence that miR-940 could be a wonderful biomarker and treatment agent for cervical cancer, and microbubble ultrasound would have more wide application in the clinical treatment of cancers.

Keywords: miR-940, microbubble, ultrasound, cell proliferation, apoptosis, cervical cancer

\section{Introduction}

Cervical cancer is one of the most common malignancies among women worldwide, and the second leading cause of women's cancer-related deaths. ${ }^{1}$ The main reason of the high mortality is cancer recurrence and metastasis. ${ }^{2}$ More than $85 \%$ of the cervical cancers occur in developing countries, causing serious damage to women's health. ${ }^{3}$ The mortality of cervical cancer in Chinese women is ranking the second place in the world, with the tendency of younger ages ( $\leq 35$ years old). ${ }^{4}$

MicroRNA (miRNA) is a kind of non-coding, small molecular RNAs, commonly regulating gene expression on post-transcriptional levels. ${ }^{5}$ Recent research 
found that miRNA played critical roles in health and disease regulation. ${ }^{6}$ The abnormal expression of miRNAs results in the occurrence and development of many cancers, including cervical cancer. ${ }^{7,8}$ Being the important reasons for tumor occurrence and development, cell cycle and cell apoptosis regulation deficiencies could be regulated by miRNAs. ${ }^{9}$ Researches on miRNA would help discovering the molecular mechanism of cancers to provide evidence for molecular diagnosis, treatment, and prognosis. ${ }^{10}$

MiR-940 has been reported as critical regulating element in various cancers. Ma et al revealed that miR-940 inhibited tumorigenesis in nasopharyngeal carcinoma cells. ${ }^{11}$ Rajendiran et al showed that miR-940 inhibited cell migration and invasion in prostate cancer. ${ }^{12}$ Yuan et al found that miR-940 was remarkably decreased in hepatocellular carcinoma tissues and cell lines. ${ }^{13}$ MiR-940 upregulation suppressed cell proliferation and induces apoptosis in ovarian cancer OVCAR3 cells. ${ }^{14}$ MiR-940 inhibited cell growth and migration in triple-negative breast cancer. ${ }^{15}$ There was clinical potential of miR-940 as a diagnostic and prognostic biomarker in breast cancer patients. ${ }^{16}$ In a previous study, Su K et al reported that miR-940 regulated p27 and PTEN post-transcriptionally to regulate human cervical cancer progression. ${ }^{17}$ Hence, we speculated miR-940 had similar tumor-inhibiting functions in cervical cancer and studied its regulation effect on cell cycle and apoptosis.

At present, the clinical application of gene treatment is not limited by ideal target genes, but lacking proper gene transfection vectors. ${ }^{18,19}$ Liposome-mediated gene transfection is widely used in labs in vitro experiments. ${ }^{20}$ But the in vivo poor targeting and low transfection efficiency limit its application in clinical gene treatment. ${ }^{21}$ Except liposome, virus vectors are of potential safety hazard by conjugating with host chromosomes, though the transfection efficiency is high. ${ }^{22}$ In addition, the poor targeting and high immunogenicity also limit its further clinical application. ${ }^{23}$ Recent researchers found that the ultrasound radiation on targeting tissues, after the injection of microbubbles with target genes, could remarkably promote the efficiency of gene transfection and expression. ${ }^{24}$

Microbubble ultrasound contrast agent is a new gene transfer vector of safe, stable, and efficient characteristics. ${ }^{25}$ The microbubble can "break" under the energy of ultrasound radiation, releasing the target gene on it. ${ }^{26}$ The vibration of microbubble destruction could increase the permeability of local cells and produce a reversible sound- hole, promoting target genes into cell nucleus to increase the efficiency of gene transfection and expression. ${ }^{27}$ Microbubbles also could protect target genes from degradation by enzymes in blood, decreasing the general side effect. ${ }^{28}$ Based on the high transfection efficiency and low side effect, the clinical application of microbubble ultrasound contrast agent in gene treatment has attracted a widespread attention. ${ }^{15}$

In our study, we studied the effect of microbubble ultrasound contrast agent for gene transfection and demonstrated the molecular mechanism of miR-940 regulating cell proliferation and apoptosis of cervical cancer Hela cells. It would provide new light for cervical cancer treatment.

\section{Materials and methods Cell culture}

Human cervical cancer Hela cells were obtained from the cell bank of Chinese Academy of Sciences (Shanghai, China) and cultured in Dulbecco's Modified Eagle Medium (DMEM, Gibco; Waltham, MA, USA) containing $10 \%$ fetal bovine serum (FBS, Gemini Bio-products, WestSacramento, CA, USA) and $1 \%$ penicillin/streptomycin (Invitrogen, USA) at $37^{\circ} \mathrm{C}$ in $5 \% \mathrm{CO}_{2}$ incubator. The medium was changed once every 2 days. Cell passage was performed when $80 \%$ cells confluent. Cells of logarithm phase were used in our research.

\section{Cell transfection}

Six fluorinated sulfur (SF6) was applied as microbubble contrast agent in this study, for the high stability, small bubble diameter and long half-life. After entering the human body, most of SF6 enters the alveoli through the lungs and is excreted through the respiratory tract, avoiding safety problem when being used. ${ }^{29}$ MiR-940 recombined pCMV plasmid and pCMV empty plasmid were purchased from Beyotime company (Nantong, China). Hela cells were divided into five groups (Control group, miR-940 group, miR-940+SF6 group, miR-940+US group and miR-940+SF6+US group). MiR-940+SF6 group and miR-940+US group were applied to exclude the effects of microbubble and ultrasound to the cells by setting two separate experimental groups for them. For miR-940 +SF6+US group, miR-940 recombined plasmid and 10\% SF6 microbubble (Bracco Suisse SA, Manno, Switzerland) were added into confluent Hela cells in serum-free media, with the ultrasonic frequency at $2.0 \mathrm{MHz}$, mechanical 
index (MI) at 0.28 and irradiation time for $30 \mathrm{~s}$. Optimal ultrasound microbubble was observed by light microscope. For miR-940+US group, miR-940 recombined plasmid was added into Hela confluent cells in serum-free media, and the ultrasound probe, accomplished by coupling agent, was used in the bottom of the culture plate, with the ultrasonic frequency at $2.0 \mathrm{MHz}, \mathrm{MI}$ at 0.28 and irradiation time for $30 \mathrm{~s}$. The temperature was monitored and kept between 36 and 37 degree. For miR-940+SF6 group, miR-940 recombined plasmid and 10\% SF6 were added into Hela confluent cells in serum-free media and conducted transfection. For miR-940 group, miR-940 recombined pCMV plasmid was transfected into Hela cells using a commonly used liposome - lipofectamine 3000 (Invitrogen, USA). At the same time, we set up a blank control group transfected with pCMV plasmid, without any other treatment, as Control group. It might be a limitation not setting up experimental groups treated with SF6 only and US only (no miR-940) as control groups. However, miR-940+SF6 group could be the control to detect the effects of US, comparing with miR-940+SF6 + US group. miR-940+US group could be the control to detect the effects of SF6, comparing with miR-940+SF6 +US group. ${ }^{18}$ After above transfections, cells were cultured with DMEM containing 10\% FBS for 6-8 hrs. Then, mRNA expression of miR-940 was detected by RT-qPCR in above groups (Control group, miR-940 group, miR-940 +SF6 group, miR-940+US group and miR-940+SF6+US group).

\section{MTT assay}

Cell viability was measured by MTT assay. Cells of different groups (Control group, miR-940 group, miR-940 +SF6 group, miR-940+US group and miR-940+SF6+US group) were seeded into 96 -well plate at $1 \times 10^{4}$ cells/well, and detected at different times $(12,24$ and $48 \mathrm{hrs})$. A total of $20 \mu \mathrm{L}$ MTT was added in each well at 12, 24 and 48 hrs, respectively, and incubated for another $4 \mathrm{hrs}$. The absorbance was quantified at $490 \mathrm{~nm}$ using the Multiskan FC microplate photometer (ThermoFisher, USA), after sediment was solved by DMSO, according to manufacturer's procedures.

\section{Apoptosis analysis}

The cell apoptosis status was detected by Annexin-V/PI (Propidium Iodide) double staining assay after $48 \mathrm{hrs}$ of transfection. Cells of different groups (Control group, miR-940 group, miR-940+SF6 group, miR-940+US group and miR-940+SF6+US group) were collected and resuspended by $500 \mu \mathrm{L}$ phosphate buffer solution (PBS, Solarbio, Beijing, China) containing $5 \mu \mathrm{L}$ Annexin-V fluorescein isothiocyanate and $5 \mu \mathrm{L}$ PI (Invitrogen, USA). After reaction for 5 mins away from light, cells were detected using BD FACS Calibur flow cytometer (BD, San Diego, CA) and the apoptosis rate was analyzed by BD CellQuestTM Pro Software version 5.1 software.

\section{Cell cycle analysis}

Cell cycle progression was evaluated by PI staining after 48 hrs of transfection. Cells of different groups (Control group, miR-940 group, miR-940+SF6 group, miR-940 +US group and miR-940+SF6+US group) were trypsinized, washed twice using PBS and fixed overnight at $4^{\circ}$ $\mathrm{C}$ in ice-cold $70 \%$ ethanol. After being washed twice with PBS again, cells were reacted with PI $(50 \mu \mathrm{g} / \mathrm{mL})$ for 30 mins at room temperature. Cell cycle analysis was immediately performed using a BD FACS Calibur flow cytometer (BD, San Diego, USA). The proportion of cells in $\mathrm{G} 1, \mathrm{~S}$ and $\mathrm{G} 2$ phases was detected.

\section{Real-time quantitative polymerase chain reaction ( $\mathrm{RT}$-qPCR)}

Quantification of miR-940 was determined by TaqMan MicroRNA Reverse Transcription Kit (Thermo Fisher, USA). The expression of miR-940 was normalized on the basis of U6. To determine mRNA levels of Puma, Bax, Bcl-2, Cleaved caspase 9, Cyclin D1 and CDK4, total RNA of different groups (Control group, miR-940 group, miR-940+SF6 group, miR-940+US group and miR940+SF6+US group) was firstly reverse transcribed by the Takara PrimeScript RT reagent kit (Takara, Japan). Quantification of mRNA was determined by TaqMan Gene Expression Assay (Thermo Fisher, USA). The preheating process was conducted for $25 \mathrm{~s}$ at $95^{\circ}$, followed by 40 cycles: denaturation at $95^{\circ} \mathrm{C}$ for $10 \mathrm{~s}$, annealing/extension at $60^{\circ} \mathrm{C}$ for $20 \mathrm{~s}$, extension at $72^{\circ} \mathrm{C}$ for $30 \mathrm{~s}$. Target gene expression was normalized by glyceraldehyde-3phosphate dehydrogenase (GAPDH). The primer sequences are listed in Table 1.

\section{Western blot}

Total protein of cells in each group was extracted using ProteoPrep ${ }^{\circledR}$ Total Extraction Sample Kit (Sigma, USA). Concentrations of proteins were tested by Bradford assay (Bio-Rad, USA), and protein samples were separated on 
Table I Primers used in RT-PCR analysis

\begin{tabular}{|l|l|l|}
\hline Name & Type & Sequence \\
\hline Puma & $\begin{array}{l}\text { Forward } \\
\text { Reverse }\end{array}$ & $\begin{array}{l}\text { AGATATTGGCGGAAGCCACC } \\
\text { CCAGATGCTCTGTCACTGGT }\end{array}$ \\
\hline Bax & $\begin{array}{l}\text { Forward } \\
\text { Reverse }\end{array}$ & $\begin{array}{l}\text { TGCTTCAGGGTTTCATCCA } \\
\text { GGCCTTGAGCACCAGTTT }\end{array}$ \\
\hline Bcl-2 & $\begin{array}{l}\text { Forward } \\
\text { Reverse }\end{array}$ & $\begin{array}{l}\text { ACGGTGGTGGAGGAGCTCTT } \\
\text { CGGTTGACGCTCTCCACAC }\end{array}$ \\
\hline Pro-caspase 9 & $\begin{array}{l}\text { Forward } \\
\text { Reverse }\end{array}$ & $\begin{array}{l}\text { CAGGCAAGCAGCAAAGTTGT } \\
\text { GCTGGTCGAAGGTCCTCAAA }\end{array}$ \\
\hline CDK4 & $\begin{array}{l}\text { Forward } \\
\text { Reverse }\end{array}$ & $\begin{array}{l}\text { GCTGGAGGTCTGCGAGGA } \\
\text { ACAGGAAGCGGTCCAGGTAGT }\end{array}$ \\
\hline GAPDH & $\begin{array}{l}\text { Forward } \\
\text { Reverse }\end{array}$ & $\begin{array}{l}\text { CTCTTTGGCAGCTGGTCACAT } \\
\text { AGGCACCGACACCAATTTCA }\end{array}$ \\
\hline
\end{tabular}

$15 \%$ sodium dodecyl sulfate-polyacrylamide gel electrophoresis (SDS-PAGE) and electroblotted to polyvinylidene fluoride (PVDF) membrane (Millipore, Billerica, USA). After the blockage with 5\% nonfat dry milk for $1 \mathrm{hr}$ at $37^{\circ} \mathrm{C}$, membranes were incubated with specific primary antibodies for Puma (Abcam, ab9643, 1:1000), Bax (Abcam, ab53154, 1:1000), Bcl-2 (Abcam ab59348, 1:1000), Cleaved caspase 9 (Abcam, ab2324, 1:1000), Cyclin D1 (Abcam, ab226977, 1:1000), and CDK4 (Abcam, ab137675, 1:2000), PI3K (Abcam, ab133595, 1:2000), AKT (Abcam, ab8805, 1:500), phosphorylated (p)-PI3K (Abcam, ab182651, 1:1000), p-Akt (Abcam, ab38449, 1:1000) and GAPDH (Abcam, ab9485, 1:2000) respectively, overnight at $4^{\circ} \mathrm{C}$. Then, they were treated with HRP (horseradish peroxidase)-conjugated secondary antibodies (Abcam, USA) at $37^{\circ} \mathrm{C}$ for $1 \mathrm{hr}$ and exposed to $\mathrm{X}$-ray film. Finally, immunoreactive bands were detected using enhanced chemiluminescence (ECL) detection reagents (Amersham, Arlington Heights, IL, USA). Finally, band densities were quantified by densitometry Bio-Rad ChemiDoc ${ }^{\mathrm{TM}}$ XRS + System with Image Lab ${ }^{\mathrm{TM}}$ Software version 4.1 (Bio-Rad Laboratories, Inc., Hercules, CA, USA).

\section{Data analysis and statistics}

Statistical analyses were performed by SPSS software (version 22.0). Data were presented as the mean \pm standard deviation from at least three independent experiments.
Turkey's test and one-way ANOVA (analysis of variance) were used in multiple groups for statistical significance. $P<0.05$ was considered statistically significant different. $P<0.01$ was considered especially significant different.

\section{Results}

\section{The transfection efficiency of miR-940 using microbubble accompanied by ultrasound in cervical cancer cells}

First of all, we conducted experiments to evaluate the effect of microbubble ultrasound transfection with miR940 on cell viabilities of Hela cells, comparing with the transfection method of liposome which was commonly used nowadays. To exclude the effect of microbubble and ultrasound to Hela cells, we set two separate experimental groups for them simultaneously. The microbubble was observed to guarantee acquiring quantities of microbubbles with good shapes (Figure 1A). The recombined miR-940 plasmids were transfected into Hela cells using different methods, respectively, involving liposome, microbubble, ultrasound and microbubble accompanied by ultrasound. The transfection efficiency was detected by RT-qPCR on miR-940 mRNA levels (Figure 1B). The miR-940 level in miR-940+SF6+US group was the highest of all, about 4.4-fold of control group transfected with pCMV empty plasmid, without any other treatment, and 2.4-fold of miR-940 group transfected by traditional liposome method. The miR-940 level of miR-940 group was 1.8-fold of control, while the miR-940 levels of miR-940 +SF6 group and miR-940+US were similar to each other, about 2.5-fold and 2.6-fold of control, respectively, which were higher than miR-940 group but lower than miR-940 +SF6+US group. There was statistically significant difference between each group $(P<0.01)$.

\section{miR-940 transfected by microbubble ultrasound inhibited cell viabilities of Hela cells}

Cell viability was detected by MTT assay. Cells of different transfection times $(12,24$ and 48 hrs) in different groups were detected. Cell viability decreased as time increased in every group transfected with miR-940. Among all groups, cell viability of miR-940+SF6+US group, about $48.0 \%$ of the Control group and $58.5 \%$ of the miR-940 group, decreased most at $48 \mathrm{hrs}(P<0.01)$. 
A

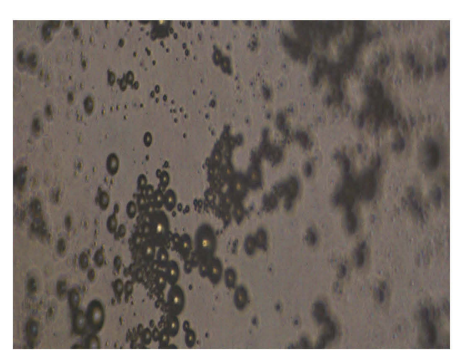

B

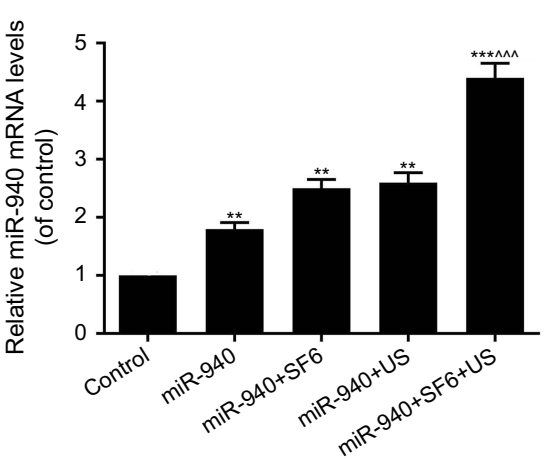

C

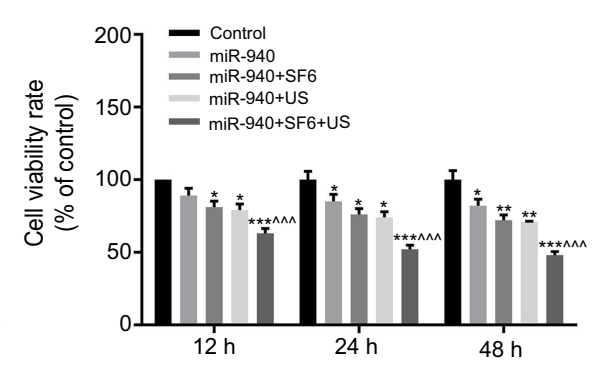

Figure I MiR-940 transfected by microbubble ultrasound inhibited cell viabilities of Hela cells. The plasmids of miR-940 were transfected into Hela cells with different methods, ie, liposome, microbubble, ultrasound and microbubble accompanied by ultrasound. The microbubble image was displayed in (A). The transfection efficiency was detected by RT-qPCR on miR-940 levels and displayed in (B). Cell viability was detected by MTT assay and displayed in (C). Cells of different transfection times (I2, 24 and 48 hrs) in different groups were detected. $* P<0.05$, $* * P<0.01$ and $* * * P<0.001$ vs control, and ${ }^{\wedge \wedge} P<0.001$ vs miR-940 group.

The viability in miR-940+SF6 group and miR-940+US were similar to each other, about $62.0 \%$ and $61.0 \%$ of the Control group, respectively, lower than miR-940 group but higher than miR-940+SF6+US group $(P<0.05)$ (Figure 1C).

\section{miR-940 transfected by microbubble ultrasound promoted cell apoptosis of Hela cells}

For the proliferation inhibition effect of miR-940 on Hela cells, we performed Annexin-V/PI double staining assay to detect the effect of miR-940, transfected by different methods, on Hela cell apoptosis. The apoptosis rate of miR-940+SF6+US group was $18.08 \%$, the highest of all, which was about 3.3-fold of control and 2.1-fold of miR940 group $(P<0.01)$. The apoptosis rate of miR-940 group $(8.57 \%)$ was 1.6 -fold of control, while that of miR-940 +SF6 group (15.82\%) and miR-940+US (16.43\%) were similar to each other, about 2.9-fold and 3.1-fold of control, respectively, higher than miR-940 group but lower than miR-940+SF6+US group ( $P<0.01$, Figure 2$)$.

\section{miR-940 transfected by microbubble ultrasound promoted cell cycle arrest of Hela cells}

For the inhibition effect of miR-940 on Hela cell proliferation, we performed PI staining assay to measure the function of miR-940, transfected by different methods, on Hela cell cycle arrest. The distribution rates of different phases were measured. As shown in Figure 3, miR-940 transfected by different methods in Hela cells blocked cell cycle transition from $\mathrm{G} 1$ to $\mathrm{S}$ phase, at different rates. The percentage of cells in G1-phase was 41.24\%, 50.79\%, $59.37 \%, 59.68 \%$ and $69.76 \%$, corresponding to control group, miR-940 group, miR-940+SF6 group, miR-940 +US group and miR-940+SF6+US group, respectively. The percentage of cells in S-phase was $24.19 \%, 19.59 \%$, $15.13 \%, 15.29 \%$ and $9.26 \%$, corresponding to control group, miR-940 group, miR-940+SF6 group, miR-940 +US group and miR-940+SF6+US group, respectively. Cell rate of miR-940+SF6+US group in G1-phase was the highest and the rate in its $\mathrm{S}$-phase was the lowest $(P<0.01$, Figure 3).

\section{miR-940 transfected by microbubble ultrasound inhibited cell viabilities of Hela cells via regulating cell cycle- and apoptosis-related factors}

To detect the mechanism of miR-940 overexpression inhibiting Hela cell viability, blocking cell cycle and promoting apoptosis, we performed RT-qPCR and Western blot to detect mRNA and protein levels of cell cycle- and apoptosis-related factors such as Puma, Bax, Bcl-2, Caspase 9, Cyclin D1 and CDK4 in the cell groups, including control group, miR-940 group, miR-940+SF6 group, miR-940 +US group and miR-940+SF6+US group. The results showed that both mRNA and protein levels of Puma and Bax increased significantly in the following order: Control group $<$ miR-940 group $<$ miR-940+SF6 group (similar to miR-940+US group) $<$ miR-940+SF6+US group $(P<0.01)$. On the contrary, both mRNA and protein levels of Bcl-2, Cleaved caspase 9, Cyclin D1 and CDK4 decreased 

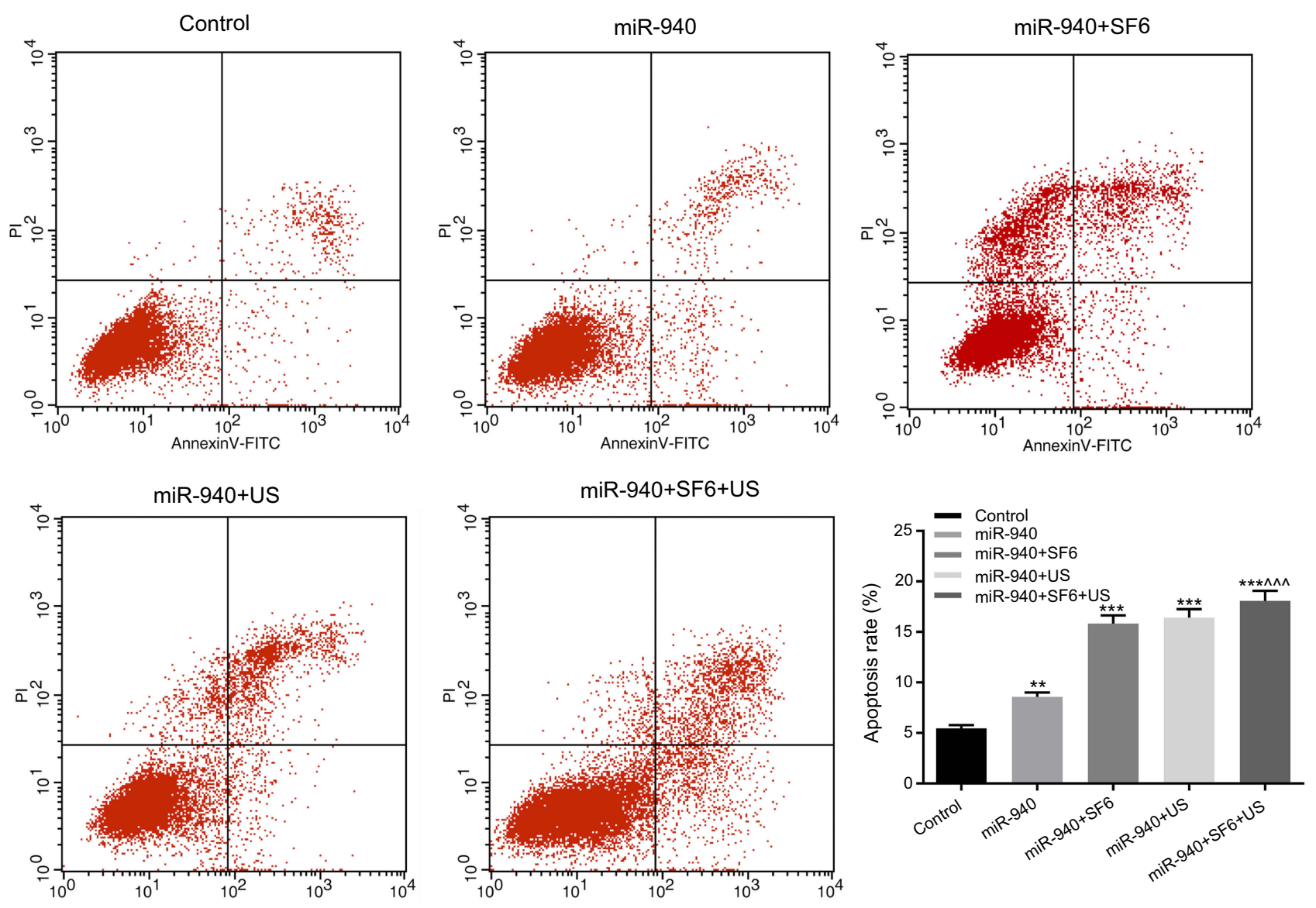

Figure 2 MiR-940 transfected by microbubble ultrasound promoted cell apoptosis of Hela cells. Annexin- V/PI double staining assay was performed to detect the effect of miR-940 transfected by different methods on Hela cell apoptosis. $* * P<0.01$ and $* * * P<0.001$ vs control, and ${ }^{\wedge \wedge} P<0.001$ vs miR-940 group.

significantly in the following order: Control group $>$ miR940 group $>$ miR-940+SF6 group (similar to miR-940+US group) $>$ miR-940+SF6+US group $(P<0.01)$. In conclusion, levels of Puma and Bax were the highest, and levels of Bcl-2, Cleaved caspase 9, Cyclin D1 and CDK4 were the lowest in miR-940+SF6+US group (Figure 4 and Figure 5).

\section{miR-940 transfected by microbubble ultrasound inhibited cell viabilities of Hela cells via inhibiting PI3K/Akt pathway}

To determine whether miR-940 regulates PI3K/Akt pathway, we performed Western blot to measure the phosphorylation levels of PI3K and Akt. The results showed that the phosphorylation levels of PI3K and Akt both decreased significantly in the following order: Control group $>$ miR940 group (similar to miR-940+SF6 group) $>$ miR-940 + US group $>$ miR-940+SF6+US group $(P<0.05)$. Phosphorylation levels of PI3K and Akt in miR-940+SF6 +US group were both the lowest among all groups.
Microbubble alone did not have too much effect on phosphorylation levels regulation, just similar to liposome transfection. But only ultrasound transfection was better than liposome transfection (Figure 6).

\section{Discussion}

Based on the high transfection efficiency and low side effect, the clinical application of microbubble ultrasound contrast agent in gene treatment has attracted a widespread attention. ${ }^{30}$ In this study, we determined the effects of different transfection methods, including liposome, microbubble, ultrasound and microbubble coupled with ultrasound, on miR-940 transfection into cervical cancer Hela cells, and the variations of cell viability, cell cycle and cell apoptosis. Though the transfection efficiency of virus vectors is also high, the potential safety hazard of conjugating with host chromosomes is also high, so we did not apply virus vector in our study. Through the research, the transfection efficiency of miR-940 transfected with liposome was the lowest and that of miR-940 transfected with 

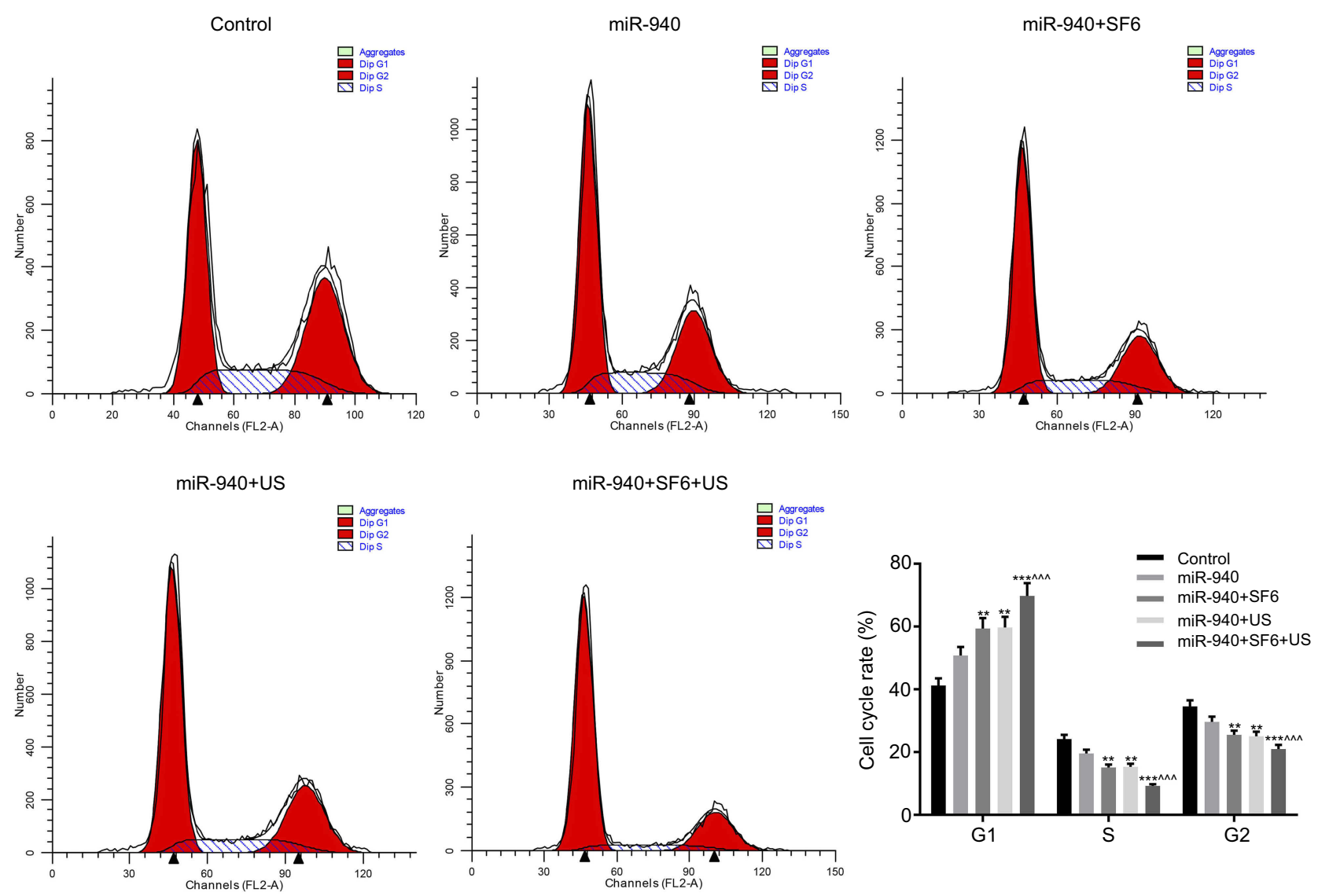

Figure 3 MiR-940 transfected by microbubble ultrasound promoted cell cycle arrest of Hela cells. PI staining assay was performed to measure the function of miR-940 transfected by different methods on Hela cell cycle arrest. $* * P<0.01$ and $* * * P<0.001$ vs control, and ${ }^{\wedge \wedge} P<0.001$ vs miR-940 group.

microbubble or ultrasonic irradiation was better than liposome. When transfected with ultrasound accompanied by microbubble, the efficiency significantly improved, better than ultrasound or microbubble alone, indicating microbubble and ultrasonic irradiation interacted to promote plasmid transfecting into cells.

The technology of microbubble ultrasound contrast agent mediated gene transfection has the incomparable advantages of high efficiency, low side effect and good targeting effect, which would be a high efficient and good targeting gene transfection system of clinical prospection. ${ }^{31}$ First, for the ultrasound could be controlled in time and space, it can break microbubble to release target genes at high concentrations in special place, realizing the targeting characteristic. ${ }^{32}$ Second, for the gas of microbubble are steady, and the short diameter and long half-life period, they can go through lung and respiratory system to be excreted to the outside of the body, therefore possessing low side effect. ${ }^{26}$ Third, the transfection efficiency is proved better than liposome. ${ }^{33}$ Microbubble ultrasound contrast agent-mediated gene transfection would be an advantageous transfection method to be widely used in the future. Ultrasound microbubble-mediated miRNA delivery into CD133+ ovarian cancer stem cells and human hepatoma HepG2 cells have been reported, and the effects were found better than the single liposome, microbubble or ultrasound, ${ }^{18,34}$ which were consistent with our research.

The emphasis of our research is on the novel transfection method of miR-940 transfected with microbubble and ultrasound. In a previous study, $\mathrm{Su} \mathrm{K}$ et al reported that miR-940 regulated $\mathrm{p} 27$ and PTEN post-transcriptionally to regulate human cervical cancer progression, ${ }^{17}$ which provided support for us using miR-940 as the target gene in our research. In our study, we successfully transfected miR-940 with microbubble and ultrasound, which promoted cervical cancer cell apoptosis, and inhibited cell proliferation significantly, the effect of which was better than the transfection of miR-940 with liposome, microbubble or ultrasound, respectively. Our study found that miR-940 overexpression inhibited Hela cell proliferation. The transfection method of ultrasound combined with microbubble enhanced the inhibition rate, which was 

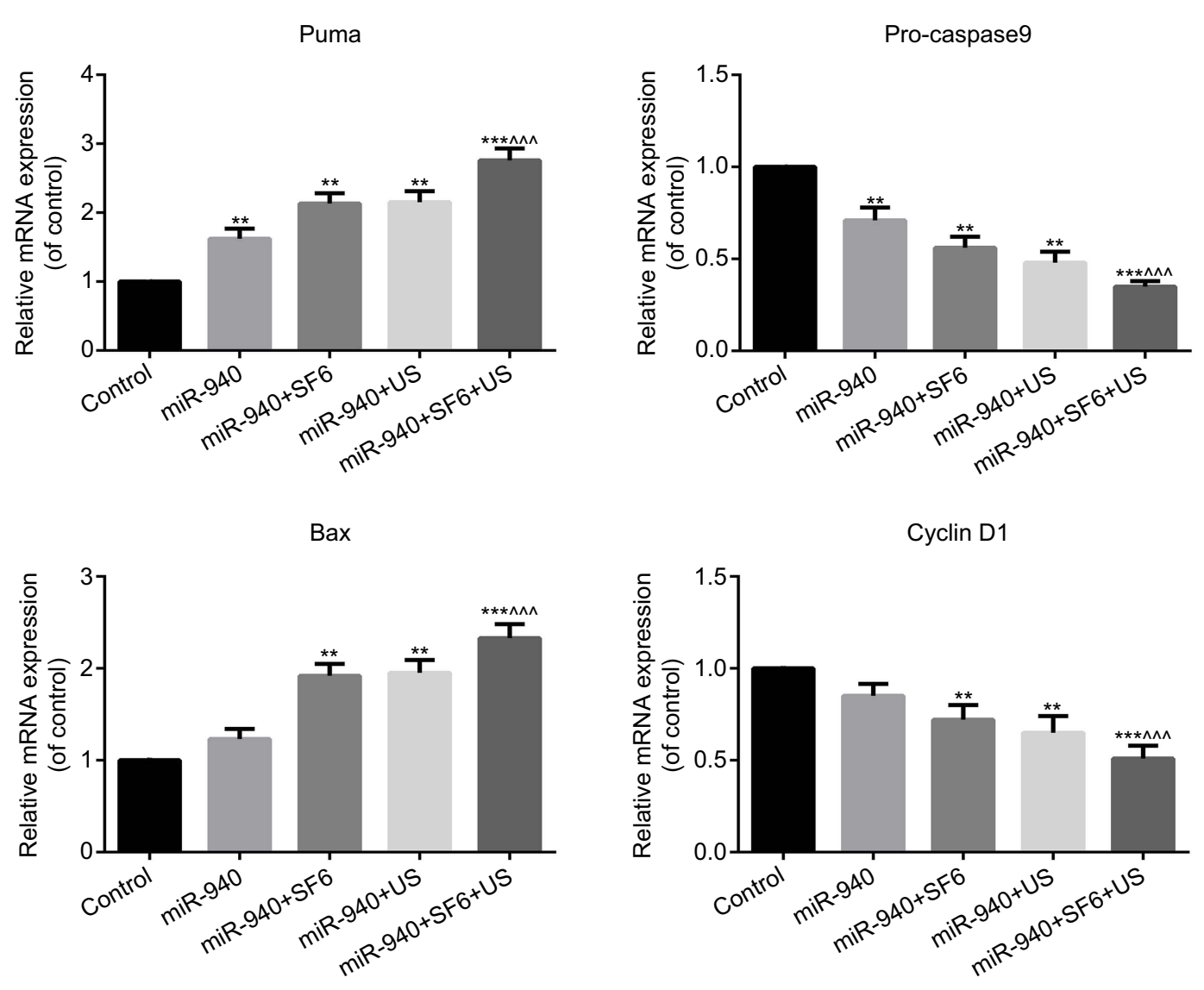

$\mathrm{Bcl}-2$
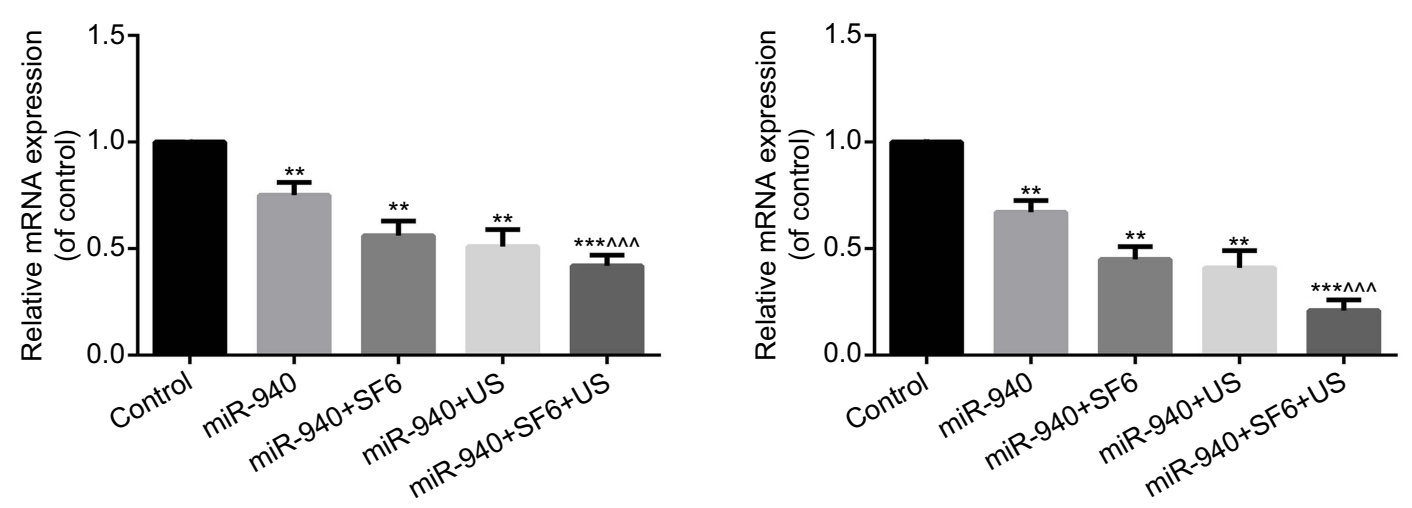

Figure 4 The mRNA levels of cell cycle- and apoptosis-related factors were regulated by miR-940, which was transfected by microbubble ultrasound. RT-qPCR was performed to detect mRNA levels of cell cycle- and apoptosis-related factors such as Puma, Bax, Bcl-2, Cleaved caspase 9, Cyclin DI and CDK4 in the cell groups, including control group, miR-940 group, miR-940+SF6 group, miR-940+US group and miR-940+SF6+US group. $* * P<0.01$ and $* * * P<0.001$ vs control, and ${ }^{\wedge \wedge} P<0.001$ vs miR-940 group.

higher than the transfection method of liposome, ultrasound or microbubble, respectively. Cell cycle and apoptosis regulation deficiency is an important reason for tumor occurrence and development. ${ }^{35}$ We found that the miR-940 overexpression transfection by ultrasound combined with microbubble could dramatically promote Hela cell apoptosis and block cell cycle from G1 to S phase, which was better than liposome, ultrasound or microbubble alone.
MiRNAs often regulates cell proliferation by modulating the critical factors in cell cycle and apoptosis, such as cyclindependent kinases (CDK), B cell lymphoma-2 (Bcl-2), Bcl-2 associated $\mathrm{X}$ protein (Bax), p53 upregulated modulator of apoptosis (Puma), PI3K/AKT pathway and so on. ${ }^{36}$ Puma, Bax, Bcl-2 and Caspase 9 are all important cell apoptosisrelated genes. ${ }^{37} \mathrm{Bcl}-2$ is proved to inhibit cell apoptosis rather than promote proliferation by regulating the outer 
A

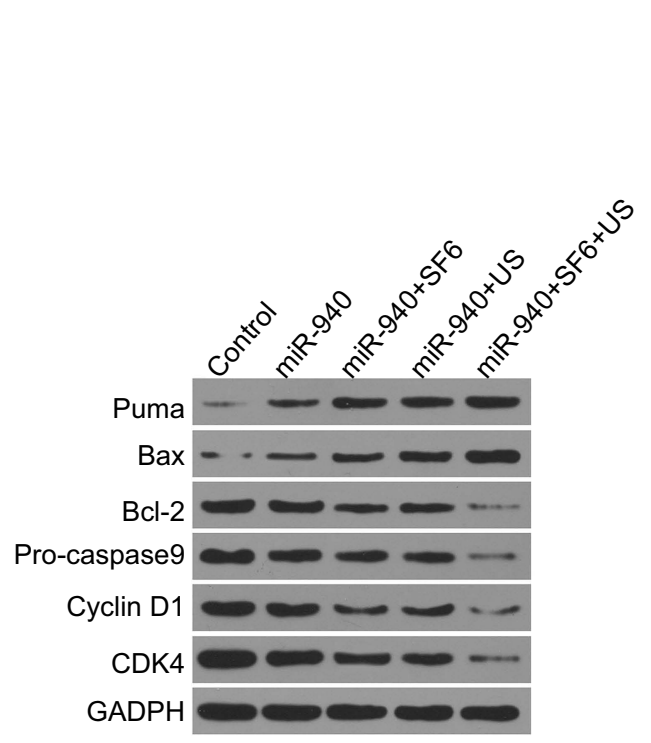

B
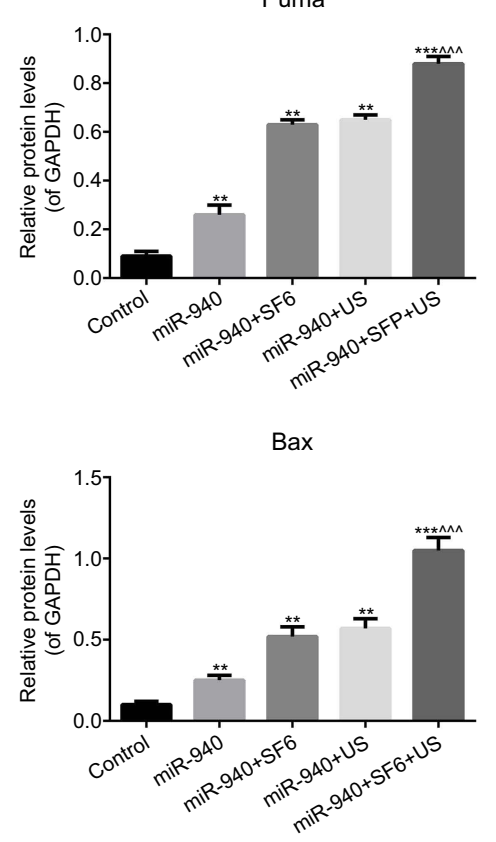

Bcl-2

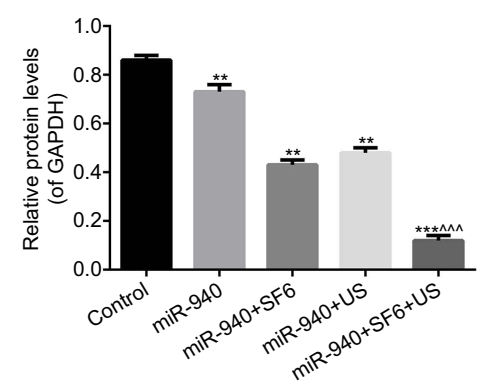

Pro-caspase9

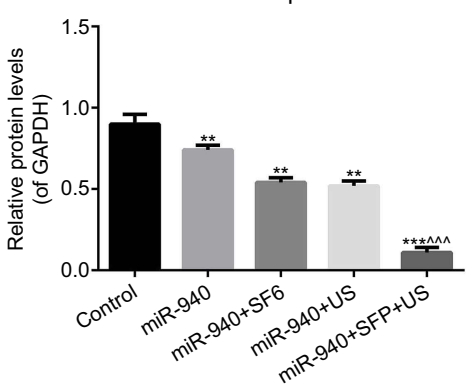

Cyclin D1

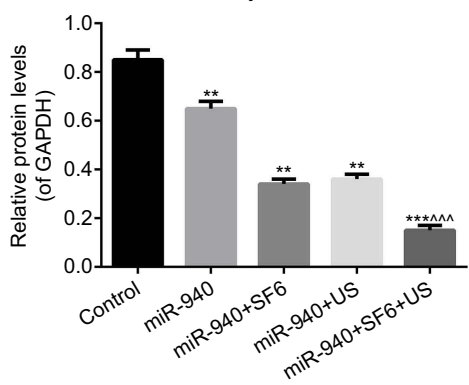

CDK4

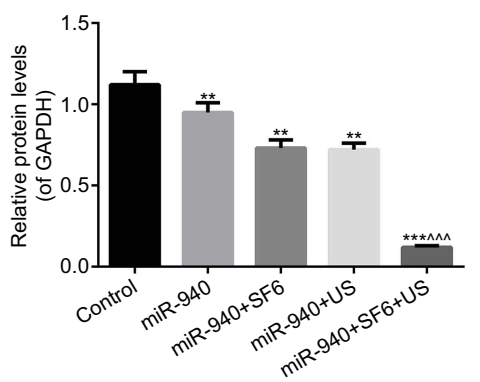

Figure 5 The protein levels of cell cycle- and apoptosis-related factors were regulated by miR-940, which was transfected by microbubble ultrasound. Western blot (A and B) was performed to detect protein levels of cell cycle- and apoptosis-related factors such as Puma, Bax, Bcl-2, Cleaved caspase 9, Cyclin DI and CDK4 in the cell groups, including control group, miR-940 group, miR-940+SF6 group, miR-940+US group and miR-940+SF6+US group. $* * P<0.01$ and $* * * P<0.001$ vs control, and ${ }^{\wedge \wedge} P<0.00 I$ vs miR940 group.

membrane permeability of mitochondria. ${ }^{38}$ Bax could form heterodimer with Bcl-2 to prevent apoptosis inhibition function of Bcl-2. ${ }^{39}$ Puma, BH3 domain-containing protein, binds to $\mathrm{Bcl}-2$ to induce cytochrome $\mathrm{c}$ release and activate cell apoptosis. $^{40,41}$ The Puma protein is also reported to promote Bax multimerization to promote apoptosis. ${ }^{42}$ Puma levels in tumor cells could be promoted by ultraviolet ray, X-radiation, chemotherapy drugs and other treatment methods. ${ }^{43}$ Caspases are intracellular proteases functioning as apoptosis initiators and effectors. Caspase 9 is critical for cytochrome c dependent apoptosis, which could be activated by Bax and inhibited by Bcl-2. ${ }^{44}$ Cleaved caspase 9 could be activated by other factors like Apaf-1, forming activated caspase 9 to activate apoptosis. ${ }^{45}$ Our study found that miR-940 overexpression transfected by ultrasound combined with microbubble increased the expression of Puma and Bax significantly, decreased Cleaved caspase 9 and the expression of apoptosis inhibition factor Bcl-2, indicating more activated caspase 9 to promote apoptosis. The changing degrees of microbubble ultrasound contrast agent group were higher than ultrasound or microbubble alone, and far more than liposome transfection.

Complexes of different Cyclins and CDKs control cell cycle progression orderly. Cyclin D1 and CDK4 are critical factors in cell cycle regulation. ${ }^{46}$ Cyclin D1 is required for cell cycle progression from G1 to S phase. ${ }^{37,47}$ The complex of CDK4 and Cyclin D1 is implicated to control cell cycle progression during G1 phase. ${ }^{48}$ Our study found that miR-940 overexpression decreased the expression of Cyclin D1 and CDK4 to inhibit cell cycle progression. The 

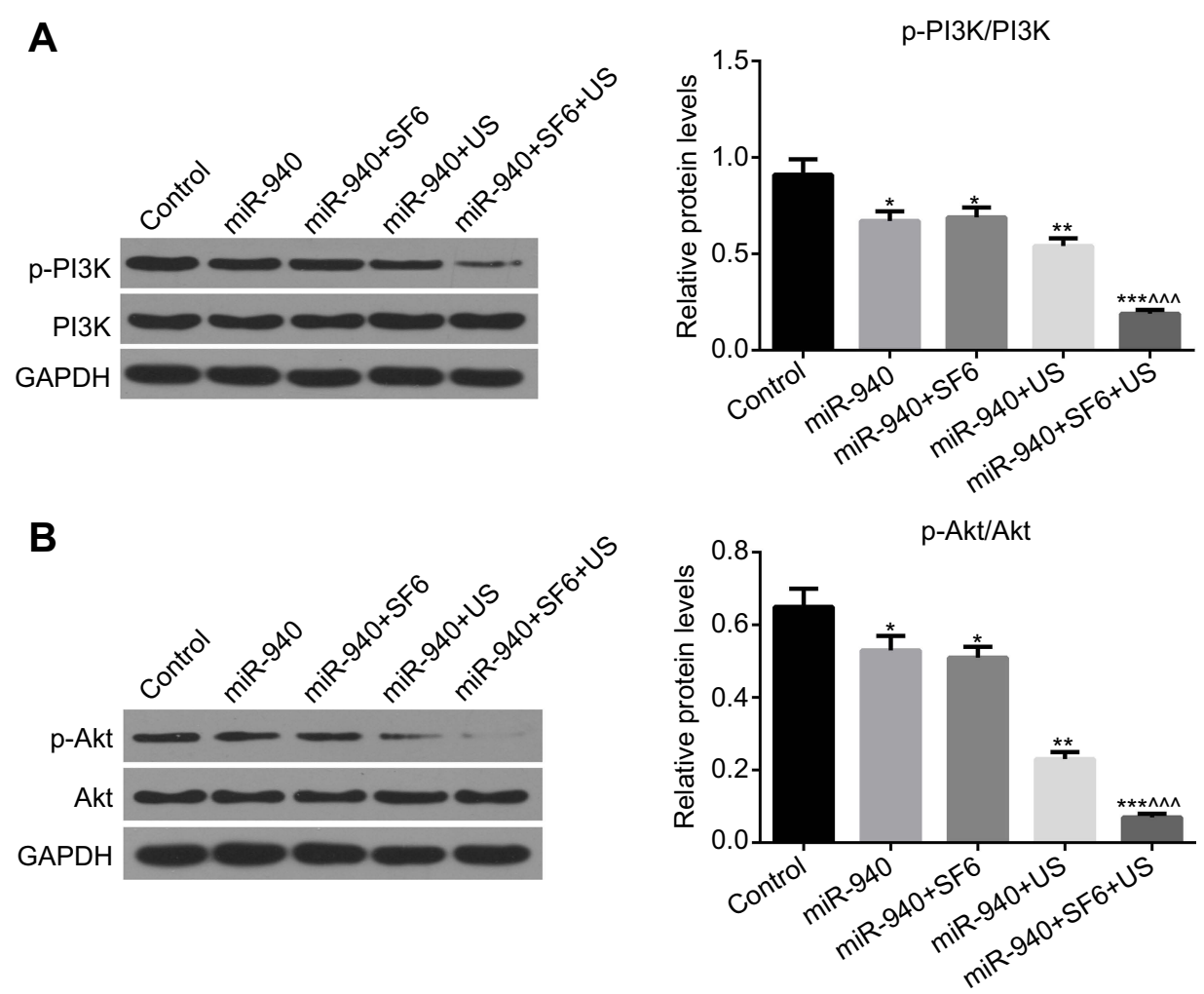

Figure 6 MiR-940 transfected by microbubble ultrasound inhibited cell viabilities of Hela cells via inhibiting PI3K/Akt pathway. Western blot was performed to measure the phosphorylation levels of $\mathrm{PI} 3 \mathrm{~K}(\mathbf{A})$ and Akt $(\mathbf{B}) . * P<0.05$, ${ }^{* * P} P<0.0 \mathrm{I}$ and $* * * P<0.00 \mathrm{I}$ vs control, and ${ }^{\wedge \wedge \wedge} P<0.00 \mathrm{I}$ vs miR-940 group.

function of the transfection method of microbubble ultrasound contrast agent was greater, compared with ultrasound, microbubble or liposome methods.

Phosphatidylinositol 3-kinase/Serine/Threonine kinase (PI3K/AKT) signaling pathway is reported to be highly related to tumorigenesis. ${ }^{49}$ The phosphorylation of Akt activates downstream target genes to regulate cell cycle, proliferation, migration and so on. ${ }^{50}$ One way is that phosphorylated Akt activates Cyclin D to promote cell cycle G1/S transition. We measured the phosphorylation levels of PI3K and Akt to determine the function of miR940 overexpression on PI3K/AKT pathway. Our study demonstrated that the phosphorylation levels of Akt and PI3K were decreased by miR-940 overexpression, and the effect of the transfection method of microbubble ultrasound contrast agent was the highest, compared with liposome, microbubble or ultrasound methods. The method of microbubble had little effect on PI3K/AKT phosphorylation. It indicated that miR-940 blocked Hela cell cycle and promoted apoptosis through decreasing phosphorylation and activation of PI3K/AKT pathway. The transfection method of microbubble with ultrasound had the greatest effect of all.

\section{Conclusion}

In conclusion, miR-940 transfected with microbubble ultrasound contrast agent is of better transfection efficiency than commonly used liposome and also better than microbubble or ultrasound, respectively. Compared with the transfection of liposome, microbubble or ultrasound, miR-940 transfected with microbubble ultrasound contrast agent showed better effects on inhibiting cell proliferation, blocking cell cycle and promoting apoptosis of cervical cancer cells, which might be associated with the regulation of cell cycle-related factors (such as Cyclin D1 and CDK4) and apoptosis-related factors (such as Puma, Bax, Bcl-2 and Cleaved caspase 9), as well as the inhibition of the phosphorylation and activation of PI3K/ AKT pathway. The research provides evidence that, for the high efficiency, low side effects and targeting characteristic, microbubble ultrasound contrast agent would have more wide application in the clinical treatment of cervical cancers. In the future, miR-940 could be a wonderful biomarker and treatment agent for cervical cancer, contributing to the diagnosis, treatment and prognosis of cervical cancer. Certainly, further research needs to be done in more aspects. 


\section{Disclosure}

The authors report no conflicts of interest in this work.

\section{References}

1. Pimple S, Mishra G, Shastri S. Global strategies for cervical cancer prevention. Curr Opin Obstet Gynecol. 2016;28(1):4-10. doi: $10.1097 /$ gco.0000000000000241

2. Henley SJ, Anderson RN, Thomas CC, Massetti GM, Peaker B, Richardson LC. Invasive cancer incidence, 2004-2013, and deaths, 2006-2015, in nonmetropolitan and metropolitan counties - United States. Morbidity and mortality weekly report. Surveill Summ. 2017;66(14):1-13. doi:10.15585/mmwr.ss6614a1

3. Beavis AL, Gravitt PE, Rositch AF. Hysterectomy-corrected cervical cancer mortality rates reveal a larger racial disparity in the United States. Cancer. 2017;123(6):1044-1050. doi:10.1002/cncr.30507

4. Bao HL, Liu YN, Wang LJ, et al. [Analysis on mortality of cervical cancer and its temporal trend in women in China [2006-2012] Zhonghua Liu Xing Bing Xue Za Zhi. 2017;38(1):58-64. doi:10.3760/cma.j.issn.0254-6450.2017.01.011

5. Song R, Cong L, Ni G, et al. MicroRNA-195 inhibits the behavior of cervical cancer tumors by directly targeting HDGF. Oncol Lett. 2017;14(1):767-775. doi:10.3892/ol.2017.6210

6. Yao J, Zhang P, Li J, Xu W. MicroRNA-215 acts as a tumor suppressor in breast cancer by targeting AKT serine/threonine kinase 1 . Oncol Lett. 2017;14(1):1097-1104. doi:10.3892/ol.2017.6200

7. Zheng Q, Cui X, Zhang D, et al. miR-200b inhibits proliferation and metastasis of breast cancer by targeting fucosyltransferase IV and alpha1,3-fucosylated glycans. Oncogenesis. 2017;6(7):e358. doi:10.10 38/oncsis. 2017.58

8. Xu N, Lian YJ, Dai X, Wang YJ. miR-7 increases cisplatin sensitivity of gastric cancer cells through suppressing mTOR. Technol Cancer Res Treat. 2017; 1533034617717863. doi:10.1177/1533034617717863

9. Mou Z, Xu X, Dong M, Xu J. MicroRNA-148b acts as a tumor suppressor in cervical cancer by inducing G1/S-phase cell cycle arrest and apoptosis in a caspase-3-dependent manner. Med Sci Monit. 2016;22:2809-2815. doi:10.12659/msm.896862

10. Macedo T, Silva-Oliveira RJ, Silva VAO, Vidal DO, Evangelista AF, Marques MMC. Overexpression of mir-183 and mir-494 promotes proliferation and migration in human breast cancer cell lines. Oncol Lett. 2017;14(1):1054-1060. doi:10.3892/ol.2017.6265

11. Ma J, Sun F, Li C, et al. Depletion of intermediate filament protein nestin, a target of microRNA-940, suppresses tumorigenesis by inducing spontaneous DNA damage accumulation in human nasopharyngeal carcinoma. Cell Death Dis. 2014;5:e1377. doi:10.1038/ cddis.2014.293

12. Rajendiran S, Parwani AV, Hare RJ, Dasgupta S, Roby RK, Vishwanatha JK. MicroRNA-940 suppresses prostate cancer migration and invasion by regulating MIEN1. Mol Cancer. 2014;13:250. doi:10.1186/1476-4598-13-250

13. Yuan B, Liang Y, Wang D, Luo F. MiR-940 inhibits hepatocellular carcinoma growth and correlates with prognosis of hepatocellular carcinoma patients. Cancer Sci. 2015;106(7):819-824. doi:10.1111/ cas. 12688

14. Wang F, Wang Z, Gu X, Cui J. miR-940 upregulation suppresses cell proliferation and induces apoptosis by targeting PKC-delta in ovarian cancer OVCAR3 cells. Oncol Res. 2017;25(1):107-114. doi:10.3727/ 096504016x14732772150145

15. Hou L, Chen M, Yang H, et al. miR-940 inhibited cell growth and migration in triple-negative breast cancer. Med Sci Monit. 2016;22:3666-3672. doi:10.12659/msm.897731

16. Liu W, Xu Y, Guan H, Meng H. Clinical potential of miR-940 as a diagnostic and prognostic biomarker in breast cancer patients. Cancer Biomark. 2018;22(3):487-493. doi:10.3233/cbm-171124
17. Su K, Wang CF, Zhang Y, Cai YJ, Zhang YY, Zhao Q. miR-940 upregulation contributes to human cervical cancer progression through p27 and PTEN inhibition. Int J Oncol. 2017. doi:10.3892/ijo.2017.3897

18. Guo X, Guo S, Pan L, Ruan L, Gu Y, Lai J. Anti-microRNA-21/221 and microRNA-199a transfected by ultrasound microbubbles induces the apoptosis of human hepatoma HepG2 cells. Oncol Lett. 2017;13 (5):3669-3675. doi:10.3892/ol.2017.5910

19. Zhou Q, Deng Q, Hu B, et al. Ultrasound combined with targeted cationic microbubble-mediated angiogenesis gene transfection improves ischemic heart function. Exp Ther Med. 2017;13(5):22932303. doi:10.3892/etm.2017.4270

20. Chen M, Zeng Z, Qu X, Tang Y, Long Q, Feng X. Biocompatible anionic polyelectrolyte for improved liposome based gene transfection. Int $J$ Pharm. 2015;490(1-2):173-179. doi:10.1016/j.ijpharm.2015.05.046

21. Kabilova T, Shmendel E, Gladkikh D, et al. Novel PEGylated liposomes enhance immunostimulating activity of isRNA. Molecules. 2018;23:12. doi:10.3390/molecules23123101

22. Du M, Chen Z, Chen Y, Li Y. Ultrasound-targeted delivery technology: A novel strategy for tumor-targeted therapy. Curr Drug Targets. 2018. doi:10.2174/1389450119666180731095441

23. Zhang M, Shi S, Guo R, Miao Y, Li B. Use of rhenium-188 for in vivo imaging and treatment of human cervical cancer cells transfected with lentivirus expressing sodium iodide symporter. Oncol Rep. 2016;36(4):2289-2297. doi:10.3892/or.2016.5034

24. Wu B, Qiao Q, Han X, et al. Targeted nanobubbles in low-frequency ultrasound-mediated gene transfection and growth inhibition of hepatocellular carcinoma cells. Tumour Biol. 2016;37(9):12113-12121. doi:10.1007/s13277-016-5082-2

25. Xie A, Wu MD, Cigarroa G, et al. Influence of DNA-microbubble coupling on contrast ultrasound-mediated gene transfection in muscle and liver. J Am Soc Echocardiogr. 2016;29(8):812-818. doi:10.1016/ j.echo.2016.04.011

26. Yamaguchi H, Ishida Y, Hosomichi J, et al. Ultrasound microbubblemediated transfection of NF-kappaB decoy oligodeoxynucleotide into gingival tissues inhibits periodontitis in rats in vivo. PLoS One. 2017;12(11):e0186264. doi:10.1371/journal.pone.0186264

27. He X, Wu DF, Ji J, Ling WP, Chen XL, Chen YX. Ultrasound microbubble-carried PNA targeting to c-myc mRNA inhibits the proliferation of rabbit iliac arterious smooth muscle cells and intimal hyperplasia. Drug Deliv. 2016;23(7):2482-2487. doi:10.3109/ 10717544.2015.1014947

28. Ji Y, Han Z, Shao L, Zhao Y. Evaluation of in vivo antitumor effects of low-frequency ultrasound-mediated miRNA-133a microbubble delivery in breast cancer. Cancer Med. 2016;5(9):2534-2543. doi:10.1002/cam4.840

29. Cheng KT. Stabilized Sulfur Hexafluoride Microbubbles. Molecular Imaging and Contrast Agent Database (MICAD). Bethesda (MD): National Center for Biotechnology Information (US; 2004.

30. Wang YJ, Zhou Q, Cao S, et al. Efficient gene therapy with a combination of ultrasoundtargeted microbubble destruction and PEI/ DNA/NLS complexes. Mol Med Rep. 2017;16(5):7685-7691. doi: $10.3892 / \mathrm{mmr} .2017 .7510$

31. Shentu W-H, Yan C-X, Liu C-M, et al. Use of cationic microbubbles targeted to P-selectin to improve ultrasound-mediated gene transfection of hVEGF 165 to the ischemic myocardium. J Zhejiang Univ Sci B. 2018;19(9):699-707. doi:10.1631/jzus.B1700298

32. Gong L, Jiang C, Liu L, et al. Transfection of neurotrophin-3 into neural stem cells using ultrasound with microbubbles to treat denervated muscle atrophy. Exp Ther Med. 2018;15(1):620-626. doi:10.3892/etm.2017.5439

33. Huang C, Zhang H, Bai R. Advances in ultrasound-targeted microbubble-mediated gene therapy for liver fibrosis. Acta Pharm Sin B. 2017;7(4):447-452. doi:10.1016/j.apsb.2017.02.004

34. Yang C, Li B, Yu J, Yang F, Cai K, Chen Z. Ultrasound microbubbles mediated miR-let-7b delivery into CD133(+) ovarian cancer stem cells. Biosci Rep. 2018;38:5. doi:10.1042/bsr20180922 
35. Lin YY, Chen JS, Wu XB, et al. Combined effects of 17beta-estradiol and exercise training on cardiac apoptosis in ovariectomized rats. PLoS One. 2018;13(12):e0208633. doi:10.1371/journal.pone.0208633

36. Park H, Huang X, Lu C, Cairo MS, Zhou X. MicroRNA-146a and microRNA-146b regulate human dendritic cell apoptosis and cytokine production by targeting TRAF6 and IRAK1 proteins. $J$ Biol Chem. 2015;290(5):2831-2841. doi:10.1074/jbc.M114.591420

37. Miyashita T, Reed JC. Tumor suppressor p53 is a direct transcriptional activator of the human bax gene. Cell. 1995;80(2):293-299. doi:10.1016/0092-8674(95)90412-3

38. Korsmeyer SJ. BCL-2 gene family and the regulation of programmed cell death. Cancer Res. 1999;59(7 Suppl):1693s-700s.

39. Tirapelli D, Lustosa IL, Menezes SB, et al. High expression of XIAP and Bcl-2 may inhibit programmed cell death in glioblastomas. Arq Neuropsiquiatr. 2017;75(12):875-880. doi:10.1590/0004-282x20170156

40. Nakano K, Vousden KH. PUMA, a novel proapoptotic gene, is induced by p53. Mol Cell. 2001;7(3):683-694.

41. Jeffers JR, Parganas E, Lee Y, et al. Puma is an essential mediator of p53-dependent and -independent apoptotic pathways. Cancer Cell. 2003;4(4):321-328.

42. Yu J, Wang Z, Kinzler KW, Vogelstein B, Zhang L. PUMA mediates the apoptotic response to p53 in colorectal cancer cells. Proc Natl Acad Sci U S A. 2003;100(4):1931-1936. doi:10.1073/pnas.2627 984100

43. Yu J, Yue W, Wu B, Zhang L. PUMA sensitizes lung cancer cells to chemotherapeutic agents and irradiation. Clin Cancer Res. 2006;12 (9):2928-2936. doi:10.1158/1078-0432.ccr-05-2429
44. Deng J, Feng J, Liu T, et al. Beraprost sodium preconditioning prevents inflammation, apoptosis, and autophagy during hepatic ischemia-reperfusion injury in mice via the P38 and JNK pathways. Drug Des Devel Ther. 2018;12:4067-4082. doi:10.2147/dddt. s182292

45. Marsden VS, O'Connor L, O'Reilly LA, et al. Apoptosis initiated by Bcl-2-regulated caspase activation independently of the cytochrome c/Apaf-1/caspase-9 apoptosome. Nature. 2002;419(6907):634-637. doi:10.1038/nature01101

46. Ingham M, Schwartz GK. Cell-Cycle Therapeutics Come of Age. $J$ Clin Oncol. 2017; Jco2016690032. doi:10.1200/jco.2016.69.0032

47. Crockford A, Zalmas LP, Gronroos E, et al. Cyclin D mediates tolerance of genome-doubling in cancers with functional p53. Ann Oncol. 2017;28(1):149-156. doi:10.1093/annonc/mdw612

48. Raspe E, Coulonval K, Pita JM, et al. CDK4 phosphorylation status and a linked gene expression profile predict sensitivity to palbociclib. EMBO Mol Med. 2017. doi:10.15252/emmm.201607084

49. Ma X, Bai Y. IGF-1 activates the P13K/AKT signaling pathway via upregulation of secretory clusterin. Mol Med Rep. 2012;6(6):14331437. doi: $10.3892 / \mathrm{mmr} .2012 .1110$

50. Liu GL, Yang HJ, Liu B, Liu T. Effects of Microrna-19b on the proliferation, apoptosis, and migration of wilms' tumor cells via the PTEN/PI3K/AKT signaling pathway. J Cell Biochem. 2017. doi:10.1002/jcb.25999

\section{Publish your work in this journal}

OncoTargets and Therapy is an international, peer-reviewed, open access journal focusing on the pathological basis of all cancers, potential targets for therapy and treatment protocols employed to improve the management of cancer patients. The journal also focuses on the impact of management programs and new therapeutic agents and protocols on patient perspectives such as quality of life, adherence and satisfaction. The manuscript management system is completely online and includes a very quick and fair peer-review system, which is all easy to use. Visit http://www.dovepress.com/ testimonials.php to read real quotes from published authors. 